\title{
Circulating renalase predicts all-cause mortality and renal outcomes in patients with advanced chronic kidney disease
}

\author{
Seon Ha Baek ${ }^{1,2}$, Ran-hui Cha ${ }^{3}$, Shin Wook Kang ${ }^{4}$, Cheol Whee Park ${ }^{5}$, Dae Ryong Cha ${ }^{6}$, Sung Gyun Kim7, \\ Sun Ae Yoon ${ }^{8}$, Sejoong Kim ${ }^{1,9}$, Sang-Youb Han ${ }^{10}$, Jung Hwan Park ${ }^{11}$, Jae Hyun Chang ${ }^{12}$, Chun Soo Lim, ${ }^{9,13}$, \\ Yon $\mathrm{Su} \mathrm{Kim}^{9,14,15}$, and Ki Young $\mathrm{Na}^{1,9}$
}

\begin{abstract}
Department of Internal Medicine, ${ }^{1}$ Seoul National University Bundang Hospital, Seongnam; ${ }^{2} \mathrm{Hallym}$ University Dongtan Sacred Heart Hospital, Hwaseong; ${ }^{3}$ National Medical Center, Seoul; ${ }^{4}$ Yonsei University College of Medicine, Seoul; ${ }^{5}$ College of Medicine, Seoul St. Mary's Hospital, The Catholic University of Korea, Seoul; ${ }^{6}$ Korea University Ansan Hospital, Ansan; ${ }^{7}$ Hallym University Sacred Heart Hospital, Anyang; ${ }^{8}$ College of Medicine, Uijeongbu St. Mary's Hospital, The Catholic University of Korea, Uijeongbu; ${ }^{9}$ Seoul National University College of Medicine, Seoul; ${ }^{10}$ Inje University Ilsan Paik Hospital, Goyang; ${ }^{11}$ Konkuk University Medical Center, Seoul; ${ }^{12}$ Gachon University Gil Medical Center, Incheon; ${ }^{13}$ Seoul Metropolitan Government Seoul National University Boramae Medical Center, Seoul; ${ }^{14}$ Department of Medical Science, Seoul National University Graduate School, Seoul; ${ }^{15}$ Kidney Research Institute, Seoul National University, Seoul, Korea
\end{abstract}

Received: February 11, 2017

Revised : April 14, 2017

Accepted: May 21, 2017
Background/Aims: Patients with chronic kidney disease (CKD) have been found to show markedly increased rates of end-stage renal disease, major adverse cardiovascular and cerebrovascular events (MACCEs), and mortality. Therefore, new biomarkers are required for the early detection of such clinical outcomes in patients with CKD. We aimed to determine whether the level of circulating renalase was associated with CKD progression, MACCEs, and all-cause mortality, using data from a prospective randomized controlled study, Kremezin STudy Against Renal disease progression in Korea (K-STAR; NCT oo860431).

Methods: A retrospective analysis of the K-STAR data was performed including 383 patients with CKD (mean age, 56.4 years; male/female, 252/131). We measured circulating renalase levels and examined the effects of these levels on clinical outcomes.

Results: The mean level of serum renalase was $75.8 \pm 34.8 \mu \mathrm{g} / \mathrm{mL}$. In the multivariable analysis, lower hemoglobin levels, higher serum creatinine levels, and diabetes mellitus were significantly associated with a higher renalase levels. Over the course of a mean follow-up period of 56 months, 25 deaths and 61 MACCEs occurred. Among 322 patients in whom these outcomes were assessed, 137 adverse renal outcomes occurred after a mean follow-up period of 27.8 months. Each 10$\mu \mathrm{g} / \mathrm{mL}$ increase in serum renalase was associated with significantly greater hazards of all-cause mortality and adverse renal outcomes (hazard ratio $[\mathrm{HR}]=1.112$, $p=0.049 ; \mathrm{HR}=1.052, p=0.045)$. However, serum renalase level was not associated with the rate of MACCEs in patients with CKD.

Conclusions: Our results indicated that circulating renalase might be a predictor of mortality and adverse renal outcomes in patients with CKD.

Keywords: Chronic kidney disease; Renalase; Mortality; End-stage renal disease

\section{INTRODUCTION}

Patients with chronic kidney disease (CKD) have been found to demonstrate markedly increased rates of mortality and cardiovascular disease (CVD); additionally, some of these patients progress to end-stage renal disease (ESRD) $[1,2]$. Therefore, it is critical to identify early and reliable biomarkers predic- 
tive of premature death, CVD, or CKD progression in patients with CKD.

Renalase is related to monoamine oxidase A, presenting 13\% amino acid identity [3-6]. The human renalase gene resides on chromosome 10 at q23.31, consists of 309,462 bp [3], and has seven tissue-specific isoforms in humans ( $\mathrm{H}$ renalase 1-7) [7]. $\mathrm{H}$ renalase 1 , the most abundant isoform, contains seven exons and encodes a 342 amino acid protein that has a molecular mass of $37.85 \mathrm{kDa}$ [3]. This isoform has been reported to be preferentially found in the glomeruli and proximal tubules, as well as in the cardiomyocytes, liver, and skeletal muscles [3,5].

Renalase is a novel protein that is secreted by the kidney, circulates in the blood, metabolizes catecholamines, and decreases cardiac contractility and systemic blood pressure in vivo [5]. In a previous study, renalase was found to be virtually undetectable in the blood of ESRD patients on hemodialysis but detectable in the blood of healthy volunteers when a Western blot analysis was performed to measure renalase concentration [5]. However, other studies have reported that increased renalase was observed in the context of reduced renal function when commercially available sandwich enzyme-linked immunosorbent assay (ELISA) kits were used [8-12]. In experimental studies, renalase deficiency has been found to be associated with increased blood pressure [13], and recombinant renalase was reported to have a hypotensive effect on blood pressure [5,13,14]. However, levels of circulating renalase have been reported to be higher in patients with hypertension than healthy individuals [3] and positively associated with blood pressure in clinical studies [9,15]. In terms of CVD, one study found renalase levels to be decreased after rats with 5/6 nephrectomized cardiac hypertrophy [16]; another study found renalase infusion to induce a lesser degree of cardiac hypertrophy and cardiac dysfunction in experimental 5/6 nephrectomized rats [17]. In one clinical study, renalase levels were higher in patients with coronary artery disease $(\mathrm{CAD})$ and correlated with decreased ejection fraction [3].

There have been discrepancies between clinical and experimental data regarding the role of renalase in blood pressure and CVD. Whether renalase may be associated with all-cause mortality and CKD progression has not yet been established, especially in a prospective study. In this study, we investigated whether renalase concen- tration was correlated with renal dysfunction and blood pressure in patients with CKD. We hypothesized that renalase might predict mortality, major adverse cardiovascular and cerebrovascular events (MACCEs), and ESRD in patients with advanced CKD. We performed the current study to determine whether renalase was associated with clinical outcomes using data from a prospective randomized controlled study, the Kremezin STudy Against Renal disease progression in Korea (K-STAR).

\section{METHODS}

\section{Study population}

Based on data from the K-STAR, a multicenter, prospective, randomized clinical trial, researchers have previously reported that the long-term use of AST-12O as an adjunct to standard treatment did not alter renal disease progression in patients with advanced CKD (Clinicaltrials.gov; NCT 00860431) [18]. A detailed study protocol has been depicted elsewhere [18]. In brief, study participants with advanced CKD were recruited from March 2009 to August 2010 and followed up for 36 months.

Table 1 summarizes the inclusion and exclusion criteria used for the K-STAR study. Of the 579 participants included in the K-STAR study, only 383 (66.1\%) who had baseline renalase data available were included in the current analyses (Fig. 1). In addition to the data obtained from the K-STAR study, we retrospectively collected data on reported survival status and MACCE occurrence through July 2015 by reviewing medical records. This retrospective expansion of the follow-up duration was also approved by the Institutional Review Boards (IRBs) of the following participating centers: Seoul National University Bundang Hospital (B-0812/066-006), Seoul National University Bundang Hospital (B-0810/oo9259), Seoul Metropolitan Government Seoul National University Boramae Medical Center (06-2009-7), Yonsei University Severance Hospital (4-2008-0586), Korea University Ansan Hospital (ASo884), Hallym University Sacred Heart Hospital (2009-Io04), Inje University Ilsan Paik Hospital (IB-o811-076), Konkuk University Medical Center (KUH1010125), and Gachon University Gil Medical Center (GIRBA2010). The need for informed consent was waived for the retrospective expansion by the IRBs of the participating centers because the study did not 
Table 1. Inclusion and exclusion criteria of K-STAR study

\begin{tabular}{|c|c|}
\hline Inclusion criteria & Exclusion criteria \\
\hline Followed over 6 months by nephrologists & Using ketosteril or AST-120 with the last 2 months \\
\hline $\begin{array}{l}\text { CKD } 3 \text { or } 4 \\
\text { eGFR by Cockcroft-Gault equation of } 15-59 \mathrm{~mL} / \mathrm{min} / 1.73 \mathrm{~m}^{2} \\
\text { Serum } \mathrm{Cr} 2.0-5.0 \mathrm{mg} / \mathrm{dL}\end{array}$ & $\begin{array}{l}\text { Gastrointestinal disease } \\
\text { Active ulcer or inflammatory bowel disease }\end{array}$ \\
\hline $\begin{array}{l}\text { Measured or expected eGFR decline of } \geq 2.5 \mathrm{~mL} / \mathrm{min} / 1.73 \mathrm{~m}^{2} \\
\text { over } 6 \text { months or } \geq 5 \mathrm{~mL} / \mathrm{min} / 1.73 \mathrm{~m}^{2} \text { over } 12 \mathrm{months}\end{array}$ & $\begin{array}{l}\text { Obstructive uropathy or reversible kidney disease } \\
\text { Autosomal polycystic kidney disease } \\
\text { Proteinuria } \geq 10 \mathrm{~g} / \text { day } \\
\text { History of kidney transplantation }\end{array}$ \\
\hline $\begin{array}{l}\text { Controlled blood pressure } \\
\text { SBP } \leq 160 \mathrm{mmHg} \text { and } \mathrm{DBP} \leq 100 \mathrm{mmHg} \\
\text { Measured three or more times at intervals of } 4 \text { weeks }\end{array}$ & $\begin{array}{l}\text { Heart failure (New York Heart Association classes } 3 \text { and 4) } \\
\text { Uncontrolled arrhythmia } \\
\text { Acute coronary syndrome } \\
\text { Cerebral infarction, hemorrhagic infarction within } 6 \text { months }\end{array}$ \\
\hline No significant changes in CKD treatment & $\begin{array}{l}\text { Active infection or uncontrolled inflammatory disease } \\
\text { Liver cirrhosis (Child-Turcotte Pugh B or C) } \\
\text { Progressive malignancy } \\
\text { Uncontrolled blood sugar (HbAic > 10\%) Hb <7.0 g/dL } \\
\text { Life expectancy < } 12 \text { months } \\
\text { Pregnancy, lactating women, planning to pregnancy }\end{array}$ \\
\hline
\end{tabular}

K-STAR, Kremezin STudy Against Renal disease progression in Korea; CKD, chronic kidney disease; eGFR, estimated glomerular filtration rate; Cr, creatinine; SBP, systolic blood pressure; DBP, diastolic blood pressure; HbAıc, hemoglobin Aıc; Hb, hemoglobin.

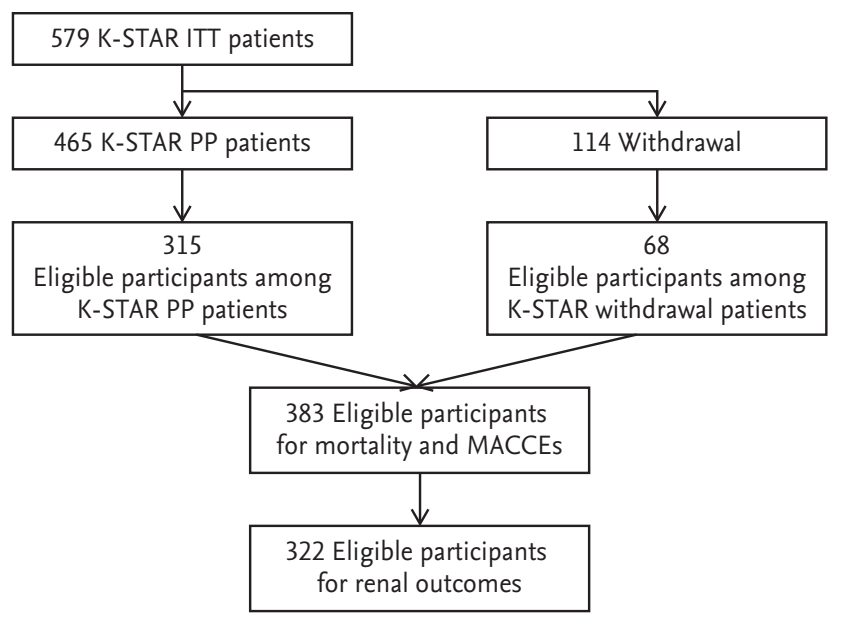

Figure 1. Study population algorithm. K-STAR, Kremezin STudy Against Renal disease progression in Korea; IT'T, intention to treat; PP, per protocol; MACCE, major adverse cardiovascular and cerebrovascular event.

infringe upon patient privacy or health status. The patients' records and data were anonymized and deidentified prior to analysis.

We further recruited 16 subjects who had not been diagnosed with CKD, hypertension, diabetes mellitus, or CVD to serve as a healthy control group and measured renalase levels in these patients to facilitate the performance of a comparison between these data and data from the K-STAR population. The collection of serum from the healthy population was also approved by the IRB of Seoul National University Bundang Hospital (B-1312/230-005) with written consent. The study was approved by the IRBs of the participating centers and conducted in accordance with the 2008 Declaration of Helsinki and good clinical practice guidelines.

\section{Data collection and definitions}

Anthropometric measurements including height and weight, and measurements of resting systolic and diastolic blood pressure were performed in the clinic. All blood samples were collected at baseline according to a standardized protocol. Serum creatinine values were measured using the alkaline picrate Jaffe kinetic method with an automatic analyzer (Toshiba-20oFR, Toshiba, Tokyo, Japan). Diabetes mellitus was confirmed based on fasting plasma glucose levels $\geq 126 \mathrm{mg} / \mathrm{dL}$, random plasma glucose $\geq 200 \mathrm{mg} / \mathrm{dL}$ with classic symptoms of hyperglycemia, 2-hour plasma glucose $\geq 200 \mathrm{mg} / \mathrm{dL}$ 
during an oral glucose tolerance test, or the use of antihyperglycemic agents or insulin.

\section{Measurement of biochemistry}

The level of serum renalase in blood samples collected at the time of K-STAR study enrollment was determined. Serum renalase concentration was measured in duplicate using the ELISA method developed by CloudClone Corp. (Katy, TX, USA). The sensitivity of the renalase assay was $1.38 \mathrm{ng} / \mathrm{mL}$, and the intra- and interassay coefficients of renalase variation were $<10 \%$ and $<$ $12 \%$, respectively.

\section{Outcomes}

The outcomes ascertained were all-cause mortality, MACCEs, and adverse renal outcomes. Data on the occurrence of death or MACCEs were obtained through a review of electronic medical records, and adverse renal outcome data were obtained from a previous randomized controlled study [18]. The MACCEs included acute coronary syndrome (ACS), heart failure, cardiac death, and stroke. ACS was defined as a clinical diagnosis of STsegment elevation myocardial infarction (STEMI), nonSTEMI, or unstable angina pectoris. ACS was confirmed based on newly identified electrocardiogram changes and/or cardiac troponin and/or typical chest pain. Coronary revascularization was defined as either coronary artery bypass grafting or percutaneous coronary intervention $[19,20]$. Heart failure was diagnosed when the patient met the appropriate Framingham criteria. Patients were required to concurrently meet two major criteria or one major plus two minor criteria be diagnosed with congestive heart failure [21]. Cardiac death was defined as mortality due to a heart-related cause (death attributable to ACS, heart failure, arrhythmia, or sudden cardiac death) [2o]. Patients were required to exhibited typical symptoms or signs of remaining neurological deficit lasting $>24$ hours with computed tomography or magnetic resonance imaging evidence of cerebral ischemic infarct or intracerebral hemorrhage to be diagnosed with stroke [22]. Adverse renal outcomes were defined as a composite outcome of serum creatinine doublings, a $>50 \%$ decrease in estimated glomerular filtration rate (eGFR), and renal replacement therapy initiation after enrollment, which matched the definition applied in the K-STAR study [18].

\section{Statistical analysis}

All analyses were conducted using IBM SPSS version 21.o (IBM Co., Armonk, NY, USA). Continuous variables were described as means \pm standard deviations, and categorical variables were presented as proportions. Differences in continuous variables were analyzed using the Student $t$ test, Mann-Whitney $U$ test, Kruskal-Wallis test, and Bonferroni's method, while chi-square tests were used to analyze differences in categorical variables. A correlation analysis was performed using Spearman correlation coefficients. Factors associated with serum renalase concentration were also evaluated via a multiple linear regression analysis. Cox proportional hazards models were used to visualize the relationships between renalase levels and all-cause mortality, MACCEs, and adverse renal outcome rates. These relationships were assessed using restricted cubic spline curves and the linearity assumption was satisfied. A backward stepwise multivariable analysis was performed to avoid co-linearity between variables. A $p<0.05$ was considered statistically significant.

\section{RESULTS}

\section{Characteristics of the study population}

A total of 383 K-STAR study participants with CKD (mean, $56.4 \pm 13.1$ years; male, 65.8\%) and 16 healthy (nondiabetic, nonhypertensive, non-CKD) controls were enrolled in the present analysis. The baseline clinical characteristics of the included 383 patients are summarized in the Supplementary Table 1. Patients with CKD had significantly higher circulating renalase concentrations than did controls (mean, $75.8 \pm 34.8$ vs. $28.2 \pm 5.1 ; p<0.001$ ). Importantly, renalase values increased significantly with increased CKD stage: controls, $28.2 \pm$ 5.1; CKD 3, $67.7 \pm$ 29.6; CKD 4, 79.7 $\pm 36.4 ; p<0.001$ (Fig. 2).

\section{Factors associated with serum renalase concentration}

In the univariable analysis, we identified diabetes mellitus, systolic blood pressure, serum creatinine, urinary protein, hemoglobin, calcium, phosphorous, and albumin levels to be significant correlated with serum renalase level. In particular, positive relationships between renalase levels and both serum creatinine levels and systolic blood pressure were observed (Fig. 3). In the 
multivariable linear regression, only hemoglobin level, high serum creatinine level, and diabetes maintained an independent association with the level of serum renalase (Table 2).

\section{Renalase and outcomes}

Over the course of a mean follow-up period of 56 months, 25 deaths and 61 MACCEs occurred. We identified a higher proportion of elderly patients in the death

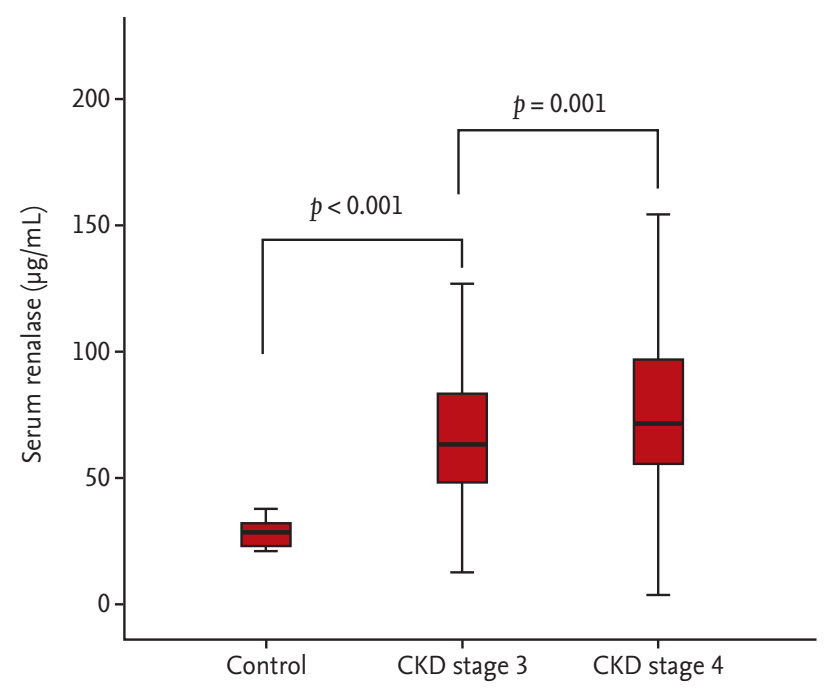

Figure 2. Serum renalase values in the control group across the chronic kidney disease (CKD) population.

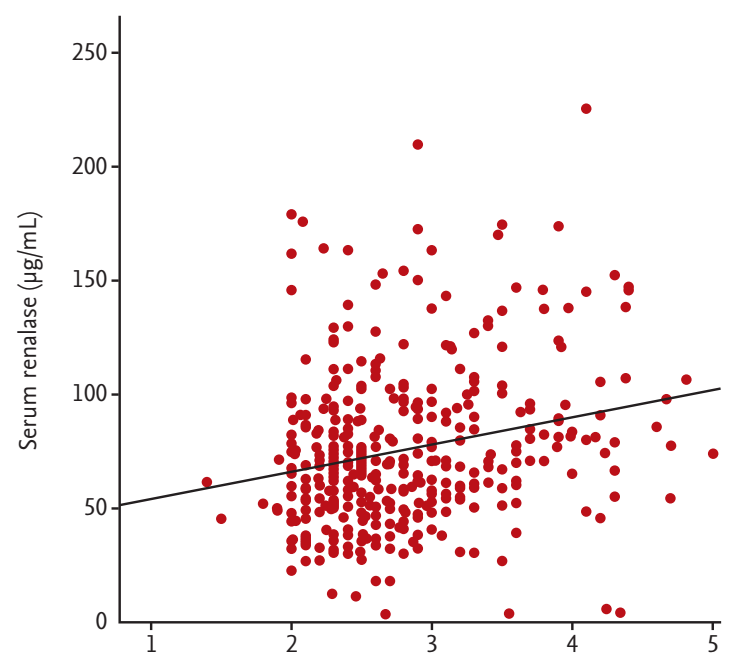

A

Serum creatinine $(\mathrm{mg} / \mathrm{dL})$ group than the survival group. Additionally, lower eGFR and hemoglobin levels and higher serum renalase levels were identified in the death group relative to the survival group (Supplementary Table 2). Each $10-\mu \mathrm{g} / \mathrm{mL}$ increase in serum renalase was associated with a significantly greater increase in the hazard of all-cause mortality (hazard ratio [HR], 1.112; $p=0.049$ ) even after adjustment for all confounders including age, sex, body mass index, systolic blood pressure, diabetic nephropathy, eGFR, urine protein to creatinine ratio, hemoglobin level, phosphorus level, albumin level, and previous randomization. However, the relationship between serum renalase level and MACCEs hazard no longer achieved statistical significance in the adjusted analysis (HR, 1.004; $p=0.910)$ (Table 3).

In the 322 patients in whom these outcomes were assessed, 137 adverse renal outcomes occurred after a mean follow-up period of 27.8 months. In the adverse renal outcomes group, a higher proportion of patients were younger and had diabetes mellitus. The adverse renal outcomes group also had lower eGFR, hemoglobin, albumin, and calcium levels and higher urinary protein, phosphorous, and renalase levels (Supplementary Table 3). Each 10- $\mu \mathrm{g} /$ $\mathrm{mL}$ increase in serum renalase concentration was associated with a significant increase in the hazard of all-cause mortality in adjusted analysis (HR, 1.052; $p=0.045)$ (Table 3$)$.

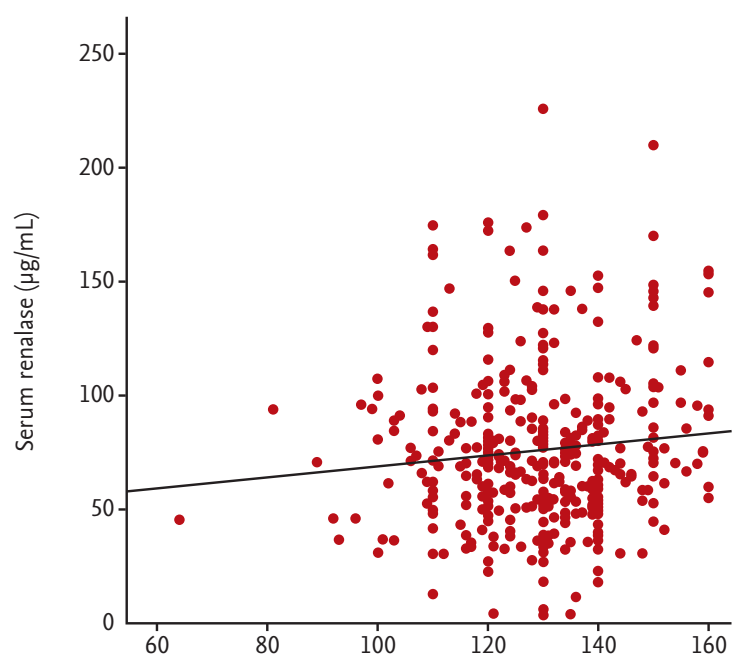

B

Systolic blood pressure $(\mathrm{mmHg})$

Figure 3. Correlations between serum renalase levels and serum creatinine (A), and systolic blood pressure (B). 


\section{DISCUSSION}

In the present study, we investigated whether renalase, which has been shown to have a contradictory impact on blood pressure and CVD in experimental and clinical studies, may function as a predictor of complications and CKD progression in patients with advanced CKD.

Table 2. Factors associates of renalase as a continuous variable in nondialysis chronic kidney disease patients

\begin{tabular}{lcr}
\hline Parameter & Standardized $\beta$ & $p$ value \\
\hline Univariable analysis & & \\
Age, yr & 0.049 & 0.338 \\
\hline Male sex, \% & -0.089 & 0.081 \\
\hline Diabetic vs. nondiabetic & 0.159 & 0.002 \\
\hline Body mass index, kg/m ${ }^{2}$ & 0.039 & 0.450 \\
\hline SBP, mmHg & 0.101 & 0.048 \\
\hline DBP, mmHg & 0.008 & 0.871 \\
\hline Serum Cr, mg/dL & 0.229 & $<0.001$ \\
\hline eGFR, mL/min/1.73m ${ }^{2}$ & -0.248 & $<0.001$ \\
\hline Urinary protein, g/g Cr & 0.128 & 0.013 \\
\hline Hemoglobin, g/dL & -0.218 & $<0.001$ \\
\hline Calcium, mg/dL & -0.146 & 0.004 \\
\hline Phosphorous, mg/dL & 0.148 & 0.004 \\
\hline Albumin, g/dL & -0.112 & 0.030 \\
\hline C-reactive protein, mg/dL & 0.083 & 0.113 \\
\hline Multivariable analysis & & 0.024 \\
\hline Hemoglobin, g/dL & -0.159 & 0.002 \\
\hline Serum Cr, mg/dL & 0.205 & $<0.001$ \\
\hline Diabetic vs. Nondiabetic & 0.116 & 0.024 \\
\hline
\end{tabular}

SBP, systolic blood pressure; DBP, diastolic blood pressure; $\mathrm{Cr}$, creatinine; eGFR, estimated glomerular filtration rate.
We found that renalase levels were higher in patients with CKD than in healthy individuals, negatively associated with eGFR, and positively associated with systolic blood pressure. The main finding of this study was that circulating renalase was a predictor of mortality and ESRD but not MACCEs in patients with CKD.

When renalase was first discovered by $\mathrm{Xu}$ et al. [5], it was a novel flavin adenine dinucleotide-dependent amine oxidase that was secreted by the kidneys, circulated in the blood, degraded catecholamines, and lowered cardiac contractility and systemic blood pressure. However, renalase shared only $13 \%$ similarities with monoamine oxidase A and B $[3,4,6]$. Furthermore, the rate of $\mathrm{H}_{2} \mathrm{O}_{2}$ synthesis measured in association with renalase was low, and its putative oxidase activity has been considered unlikely to have physiologic significance [23-25]. A recent study showed that renalase promoted cell survival and protected against toxic and ischemic acute kidney injury via the activation of intracellular signaling and independent of its ability to metabolize catecholamines [23]. However, as mentioned above, the biochemical function of renalase remains poorly understood.

To clarify the clinical role of renalase in patients with CKD, we first intended to determine whether renalase concentration was correlated with eGFR and blood pressure, as has been previously reported. In accordance with the results of other clinical studies, our results showed that circulating renalase levels were higher in patients with CKD than in healthy individuals and were negatively associated with eGFR [8-12]. The renalase levels identified in our study were similar to those reported in other studies that used the same ELISA kit for re-

Table 3. Multivariable Cox analysis predicting all-cause mortality and major adverse cardiovascular and cerebrovascular events and chronic kidney disease progression

\begin{tabular}{|c|c|c|c|c|c|c|}
\hline \multirow{2}{*}{$\times 10$} & \multicolumn{2}{|c|}{ Univariable } & \multicolumn{2}{|c|}{ Multivariable $^{\mathrm{a}}$} & \multicolumn{2}{|c|}{ Multivariable $^{\mathrm{b}}$} \\
\hline & $\operatorname{HR}(95 \% \mathrm{CI})$ & $p$ value & $\operatorname{HR}(95 \% \mathrm{CI})$ & $p$ value & $\operatorname{HR}(95 \% \mathrm{CI})$ & $p$ value \\
\hline All-cause mortality & $1.123(1.027-1.228)$ & 0.011 & $1.119(1.017-1.232)$ & 0.021 & $1.112(1.000-1.236)$ & 0.049 \\
\hline MACCEs & $1.054(0.987-1.126)$ & 0.113 & $1.046(0.977-1.119)$ & 0.195 & $1.004(0.929-1.086)$ & 0.910 \\
\hline Renal outcome & $1.070(1.024-1.117)$ & 0.002 & $1.071(1.026-1.118)$ & 0.002 & $1.052(1.001-1.105)$ & 0.045 \\
\hline
\end{tabular}

HR, hazard ratio; CI, confidence interval; MACCE, major adverse cardiovascular and cerebrovascular event.

${ }^{\text {a Age and sex. }}$

${ }^{\mathrm{b}}$ Age, sex, body mass index, systolic blood pressure, diabetic nephropathy, estimated glomerular filtration rate, urine protein creatinine ratio, hemoglobin, phosphorous, previous randomization, and albumin. 
nalase measurement (Cloud-Clone Corp.) [3,8,10,26-29]. The positive relationship between renalase and blood pressure that was observed in our data was also in accordance with the results of some other clinical studies [9,15]; however, recombinant renalase, in particular, has been shown to have a hypotensive effect on blood pressure in experimental studies $[5,13,14]$.

Whether renalase can be a predictor for CKD complications or CKD progression has not previously been reported, especially in nondialyzed patients with advanced CKD. To our knowledge, this is the first study to identify the prognostic usefulness of renalase in patients with CKD using data from a prospective, randomized controlled study. Positive associations were observed between baseline renalase level and mortality/ESRD in our study. We demonstrated that although renalase may serve as a novel prognostic biomarker for all-cause mortality and ESRD, even after adjustment for potential covariates including previous randomization, it was not predictive of the occurrence of MACCEs in patients with CKD. Available data on renalase in CVD have been derived predominantly from experimental models of heart failure. Studies have suggested that renalase levels decreased in rats with cardiac hypertrophy after 5/6 nephrectomy [16], and renalase infusion induced a lesser degree of cardiac hypertrophy and cardiac dysfunction in 5/6 nephrectomized rats [17]. Impaired synthesis of renalase may represent a mechanism potentially underlying the accumulation of circulating catecholamines in heart failure [30]. Previous clinical studies reporting the effect of renalase on CVD have been relatively small in scale. One small-scale cross-section study reported that NT-proBNP levels were inversely correlated with renalase levels [31]. In contrast, a different study including 121 patients with primary hypertension reported that renalase levels were higher in patients with CAD and correlated with a decreased ejection fraction [3]. Among dialysis patients, higher renalase levels were also observed in patients with $\mathrm{CAD}$ and in the cardiac death group than in the noncardiac death group [8].

As noted above, several controversial issues regarding the physiologic role of renalase remain. In actuality, we cannot prove with certainty whether renalase was a causative factor or a bystander in terms of mortality or ESRD because of the observational nature of the study. We considered possible reasons for the observed nega- tive effect of renalase on survival and renal outcomes in patients with CKD. First, renalase levels could be elevated because of not only decreases in the renal clearance of renalase but also catecholamine excess or activation of the sympathetic nerve system in CKD or ESRD [32,33]. Plasma levels of norepinephrine have been reported to be elevated in CKD, leading to increased sympathetic nerve activity [32]. Li et al. [6] reported that infusion of catecholamines caused an increase in blood renalase activity in experimental animals. Gu et al. [30] also reported that renalase protein levels peaked at 1 week, declining thereafter to reach subbasal levels after left anterior descending artery ligation under experimental condition. The authors proposed that the kidney might synthesize and secrete additional renalase to compensate for increases in catecholamine levels during the early phase of cardiac function deterioration, hypothesizing that as cardiac function continues to deteriorate, the expression of renalase by the kidney fails to keep pace with the increase in catecholamines, resulting in a phase of decompensation. In other words, elevated renalase levels might reflect elevated catecholamine levels, which are known to be predictive of both survival and CKD progression in patients with CKD [34].

Our study had several limitations. First, our sample size and number of outcomes analyzed were ultimately too small to have sufficient statistical power. Second, we did not estimate the levels of catecholamines such as dopamine and norepinephrine. Therefore, we could not explore relationship between catecholamine and renalase levels, or the synergistic or antagonistic effects of catecholamines and renalase on clinical outcomes.

In conclusion, our data indicate that the level of circulating renalase was predictive of all-cause mortality and ESRD, but not MACCEs in patients with CKD.

\section{KEY MESSAGE}

1. Renalase levels were higher in patients with chronic kidney disease (CKD) than in healthy individuals, negatively associated with estimated glomerular filtration rate, and were positively associated with systolic blood pressure.

2. The level of circulating renalase was predictive of mortality and end-stage renal disease outcomes, but not major adverse cardiovascular and cerebrovascular events in patients with CKD. 


\section{Conflict of interest}

No potential conflict of interest relevant to this article was reported.

\section{Acknowledgments}

The authors would like to thank Soyeon Ahn, PhD (Medical Research Collaborating Center, Seoul National University Bundang Hospital) for valuable comments of statistical methods without compensation.

\section{REFERENCES}

1. Spasovski G, Ortiz A, Vanholder R, El Nahas M. Proteomics in chronic kidney disease: the issues clinical nephrologists need an answer for. Proteomics Clin Appl 2011;5:233-240.

2. Barsoum RS. Chronic kidney disease in the developing world. N Engl J Med 2006;354:997-999.

3. Maciorkowska D, Zbroch E, Malyszko J. Circulating renalase, catecholamines, and vascular adhesion protein 1 in hypertensive patients. J Am Soc Hypertens 2015;9:855864.

4. Desir GV, Wang L, Peixoto AJ. Human renalase: a review of its biology, function, and implications for hypertension. J Am Soc Hypertens 2012;6:417-426.

5. Xu J, Li G, Wang P, et al. Renalase is a novel, soluble monoamine oxidase that regulates cardiac function and blood pressure. J Clin Invest 2005;115:1275-1280.

6. Li G, Xu J, Wang P, et al. Catecholamines regulate the activity, secretion, and synthesis of renalase. Circulation 2008;117:1277-1282.

7. Guo X, Wang L, Velazquez H, Safirstein R, Desir GV. Renalase: its role as a cytokine, and an update on its association with type 1 diabetes and ischemic stroke. Curr Opin Nephrol Hypertens 2014;23:513-518.

8. Zbroch E, Koc-Zorawska E, Malyszko J, Malyszko J, Mysliwiec M. Circulating levels of renalase, norepinephrine, and dopamine in dialysis patients. Ren Fail 2013;35:673679.

9. Wang F, Li J, Xing T, Xie Y, Wang N. Serum renalase is related to catecholamine levels and renal function. Clin Exp Nephrol 2015;19:92-98.

10. Przybylowski P, Malyszko J, Kozlowska S, Malyszko J, KocZorawska E, Mysliwiec M. Serum renalase depends on kidney function but not on blood pressure in heart trans- plant recipients. Transplant Proc 2011;43:3888-3891.

11. Malyszko J, Zbroch E, Malyszko JS, Koc-Zorawska E, Mysliwiec M. Renalase, a novel regulator of blood pressure, is predicted by kidney function in renal transplant recipients. Transplant Proc 2011;43:3004-3007.

12. Gluba-Brzozka A, Michalska-Kasiczak M, Franczyk-Skora B, Nocun M, Banach M, Rysz J. Markers of increased cardiovascular risk in patients with chronic kidney disease. Lipids Health Dis 2014;13:135.

13. Desir GV, Tang L, Wang P, et al. Renalase lowers ambulatory blood pressure by metabolizing circulating adrenaline. J Am Heart Assoc 2012;1:e002634.

14. Desir GV. Role of renalase in the regulation of blood pressure and the renal dopamine system. Curr Opin Nephrol Hypertens 2011;20:31-36.

15. Wang F, Huang B, Li J, Liu L, Wang N. Renalase might be associated with hypertension and insulin resistance in type 2 diabetes. Ren Fail 2014;36:552-556.

16. Ghosh SS, Krieg RJ, Sica DA, Wang R, Fakhry I, Gehr T. Cardiac hypertrophy in neonatal nephrectomized rats: the role of the sympathetic nervous system. Pediatr Nephrol 2009;24:367-377.

17. Baraka A, El Ghotny S. Cardioprotective effect of renalase in 5/6 nephrectomized rats. J Cardiovasc Pharmacol Ther 2012;17:412-416.

18. Cha RH, Kang SW, Park CW, et al. A randomized, controlled trial of oral intestinal sorbent AST-120 on renal function deterioration in patients with advanced renal dysfunction. Clin J Am Soc Nephrol 2016;11:559-567.

19. Cheng JM, Akkerhuis KM, Meilhac O, et al. Circulating osteoglycin and NGAL/MMP 9 complex concentrations predict 1-year major adverse cardiovascular events after coronary angiography. Arterioscler Thromb Vasc Biol 2014;34:1078-1084.

20. Muhlestein JB, Lappe DL, Lima JA, et al. Effect of screening for coronary artery disease using CT angiography on mortality and cardiac events in high-risk patients with diabetes: the FACTOR-64 randomized clinical trial. JAMA 2014;312:2234-2243.

21. Ho KK, Pinsky JL, Kannel WB, Levy D. The epidemiology of heart failure: the Framingham Study. J Am Coll Cardiol 1993;22(4 Suppl A):6A-13A.

22. Westin GG, Armstrong EJ, Bang H, et al. Association between statin medications and mortality, major adverse cardiovascular event, and amputation-free survival in patients with critical limb ischemia. J Am Coll Cardiol 
2014;63:682-69o.

23. Wang L, Velazquez H, Moeckel G, et al. Renalase prevents AKI independent of amine oxidase activity. J Am Soc Nephrol 2014;25:1226-1235.

24. Milani M, Ciriello F, Baroni S, et al. FAD-binding site and NADP reactivity in human renalase: a new enzyme involved in blood pressure regulation. J Mol Biol 2011;411:463-473.

25. Boomsma F, Tipton KF. Renalase, a catecholamine-metabolising enzyme? J Neural Transm (Vienna) 2007;114:775776.

26. Malyszko J, Koc-Zorawska E, Zorawski M, et al. Renalase is removed by kidneys and during dialysis: excess related to CKD complications? Curr Vasc Pharmacol 2015;13:134140.

27. Koc-Zorawska E, Malyszko J, Malyszko JS, Mysliwiec M. VAP-1, a novel molecule linked to endothelial damage and kidney function in kidney allograft recipients. Kidney Blood Press Res 2012;36:242-247.

28. Wybraniec MT, Mizia-Stec K, Trojnarska O, et al. Low plasma renalase concentration in hypertensive patients after surgical repair of coarctation of aorta. J Am Soc Hypertens 2014;8:464-474.

29. Zbroch E, Malyszko J, Malyszko JS, Koc-Zorawska E, Mys- liwiec M. Renalase, a novel enzyme involved in blood pressure regulation, is related to kidney function but not to blood pressure in hemodialysis patients. Kidney Blood Press Res 2012;35:395-399.

30. Gu R, Lu W, Xie J, Bai J, Xu B. Renalase deficiency in heart failure model of rats: a potential mechanism underlying circulating norepinephrine accumulation. PLoS One 2011;6:e14633.

31. Dziedzic M, Petkowicz B, Bednarek-Skublewska A, Solski J, Buczaj A, Choina P. Relationship between renalase and N-terminal pro-B-type natriuretic peptide (NT proBNP) in haemodialysis patients. Ann Agric Environ Med 2014;21:132-135.

32. Malyszko J, Malyszko JS, Mikhailidis DP, Rysz J, Zorawski M, Banach M. Hypertension and kidney disease: is renalase a new player or an innocent bystander? J Hypertens 2012;30:457-462.

33. Eisenhofer G, Rundquist B, Aneman A, et al. Regional release and removal of catecholamines and extraneuronal metabolism to metanephrines. J Clin Endocrinol Metab 1995;80:3009-3017.

34. Schlaich MP, Socratous F, Hennebry S, et al. Sympathetic activation in chronic renal failure. J Am Soc Nephrol 2009;20:933-939. 
Supplementary Table 1. Baseline characteristics at initiation of study

\begin{tabular}{lcc}
\hline Characteristic & Number & Value \\
\hline Age, yr & 383 & $56.4 \pm 13.1$ \\
Male sex & 383 & $252(65.8)$ \\
ESRD cause & 383 & \\
$\quad$ Diabetic & & $162(47.1)$ \\
$\quad$ Non-diabetic & & $182(52.9)$ \\
Body mass index, kg/m ${ }^{2}$ & 383 & $24.6 \pm 3.6$ \\
Systolic blood pressure, mmHg & 382 & $129.5 \pm 14.6$ \\
Diastolic blood pressure, $\mathrm{mmHg}$ & 382 & $75.7 \pm 9.9$ \\
\hline Serum Cr, mg/dL & 383 & $2.82 \pm 0.67$ \\
eGFR, mL/min/1.73 m ${ }^{2}$ & 383 & $26.84 \pm 7.19$ \\
\hline Urinary protein, g/g Cr & 375 & $2.11 \pm 2.32$ \\
Hemoglobin, g/dL & 382 & $11.4 \pm 1.8$ \\
\hline Albumin, g/dL & 379 & $3.99 \pm 0.45$ \\
\hline Uric acid, mg/dL & 382 & $8.34 \pm 1.88$ \\
\hline LDL, mg/dL & 327 & $92.4 \pm 34.6$ \\
C-reactive protein, mg/dL & 365 & $0.67 \pm 2.71$ \\
Renalase, $\mu$ g/mL & 383 & $75.8 \pm 34.8$ \\
\hline
\end{tabular}

Values are presented as mean \pm SD or number (\%).

ESRD, end stage renal disease; Cr, creatinine; eGFR, estimated glomerular filtration rate; LDL, low density lipoprotein. 
Supplementary Table 2. Baseline characteristics of patients according to the occurrence of death in 383 patients

\begin{tabular}{|c|c|c|c|}
\hline Variable & No death $\left(n=35^{8}\right)$ & Death $(n=25)$ & $p$ value \\
\hline Age, yr & $55.8 \pm 13.0$ & $65.9 \pm 10.7$ & $<0.001$ \\
\hline Male sex & $236(65 \cdot 9)$ & $16(64.0)$ & 0.830 \\
\hline \multicolumn{4}{|l|}{ ESRD cause } \\
\hline Diabetic & $178(49.7)$ & $17(68.0)$ & 0.098 \\
\hline BMI, $\mathrm{kg} / \mathrm{m}^{2}$ & $24.7 \pm 3.7$ & $23.9 \pm 2.6$ & 0.285 \\
\hline $\mathrm{SBP}, \mathrm{mmHg}$ & $129.2 \pm 14.5$ & $133.2 \pm 16.0$ & 0.199 \\
\hline $\mathrm{DBP}, \mathrm{mmHg}$ & $75.5 \pm 10.0$ & $78.0 \pm 8.1$ & 0.242 \\
\hline Serum Cr, mg/dL & $2.80 \pm 0.66$ & $3.00 \pm 0.79$ & 0.163 \\
\hline $\mathrm{eGFR}, \mathrm{mL} / \mathrm{min} / 1.73 \mathrm{~m}^{2}$ & $27.15 \pm 7.12$ & $22.24 \pm 6.60$ & 0.001 \\
\hline Urinary protein, g/g Cr & $2.12 \pm 2.34$ & $1.88 \pm 2.11$ & 0.617 \\
\hline Hemoglobin, g/dL & $11.4 \pm 1.7$ & $10.4 \pm 1.7$ & 0.005 \\
\hline Albumin, g/dL & $4.0 \pm 0.4$ & $3.9 \pm 0.5$ & 0.243 \\
\hline Uric acid, mg/dL & $8.4 \pm 1.9$ & $8.0 \pm 1.9$ & 0.396 \\
\hline $\mathrm{LDL}, \mathrm{mg} / \mathrm{dL}$ & $92.5 \pm 35.5$ & $91.2 \pm 20.2$ & 0.852 \\
\hline $\mathrm{CRP}, \mathrm{mg} / \mathrm{dL}$ & $0.68 \pm 2.80$ & $0.52 \pm 0.96$ & 0.788 \\
\hline Calcium, mg/dL & $8.87 \pm 0.57$ & $8.78 \pm 0.74$ & 0.476 \\
\hline Phosphorous, mg/dL & $3.89 \pm 0.73$ & $3.88 \pm 0.83$ & 0.962 \\
\hline Renalase, $\mu \mathrm{g} / \mathrm{mL}$ & $74.7 \pm 34.2$ & $92.4 \pm 40.3$ & 0.014 \\
\hline Follow-up period, mon & $57.8 \pm 14.8$ & $34.2 \pm 17.4$ & 0.004 \\
\hline
\end{tabular}

Values are presented as mean \pm SD or number (\%).

ESRD, end stage renal disease; BMI, body mass index; SBP, systolic blood pressure; DBP, diastolic blood pressure; Cr, creatinine; eGFR, estimated glomerular filtration rate; LDL, low density lipoprotein; CRP, C-reactive protein. 
Supplementary Table 3. Baseline characteristics of patients according to the occurrence of renal outcomes in 322 patients

\begin{tabular}{|c|c|c|c|}
\hline Variable & No renal outcome $(n=185)$ & Renal outcome $(n=137)$ & $p$ value \\
\hline Age, yr & $57.6 \pm 13.3$ & $54.1 \pm 12.7$ & 0.016 \\
\hline Male sex & $121(65.4)$ & $90(65 \cdot 7)$ & 1.000 \\
\hline \multicolumn{4}{|l|}{ ESRD cause } \\
\hline Diabetic & $80(43.2)$ & $82(59.9)$ & 0.003 \\
\hline BMI, $\mathrm{kg} / \mathrm{m}^{2}$ & $24.8 \pm 3.7$ & $24 \cdot 3 \pm 3 \cdot 4$ & 0.242 \\
\hline $\mathrm{SBP}, \mathrm{mmHg}$ & $128.5 \pm 15.0$ & $130.6 \pm 14.0$ & 0.212 \\
\hline $\mathrm{DBP}, \mathrm{mmHg}$ & $75.1 \pm 9.6$ & $76.2 \pm 9.9$ & 0.292 \\
\hline Serum Cr, mg/dL & $2.60 \pm 0.55$ & $3.03 \pm 0.69$ & $<0.001$ \\
\hline $\mathrm{eGFR}, \mathrm{mL} / \mathrm{min} / 1 \cdot 73 \mathrm{~m}^{2}$ & $28.29 \pm 7.26$ & $25.21 \pm 5.95$ & $<0.001$ \\
\hline Urinary protein, g/g Cr & $1.27 \pm 1.54$ & $3.18 \pm 2.21$ & $<0.001$ \\
\hline Hemoglobin, g/dL & $11.8 \pm 1.8$ & $10.9 \pm 1.5$ & $<0.001$ \\
\hline Albumin, g/dL & $4.1 \pm 0.4$ & $3.8 \pm 0.5$ & $<0.001$ \\
\hline Uric acid, mg/dL & $8.3 \pm 1.8$ & $8.3 \pm 1.8$ & 0.749 \\
\hline $\mathrm{LDL}, \mathrm{mg} / \mathrm{dL}$ & $91.6 \pm 26.0$ & $93.1 \pm 33.3$ & 0.677 \\
\hline $\mathrm{CRP}, \mathrm{mg} / \mathrm{dL}$ & $0.69 \pm 2.65$ & $0.61 \pm 3.09$ & 0.823 \\
\hline Calcium, mg/dL & $9.00 \pm 0.55$ & $8.72 \pm 0.59$ & $<0.001$ \\
\hline Phosphorous, mg/dL & $3.75 \pm 0.70$ & $4.10 \pm 0.69$ & $<0.001$ \\
\hline Renalase, $\mu \mathrm{g} / \mathrm{mL}$ & $69.6 \pm 31.8$ & $84.0 \pm 35.8$ & $<0.001$ \\
\hline Follow-up period, mon & $32.4 \pm 4.2$ & $21.5 \pm 8.5$ & $<0.001$ \\
\hline
\end{tabular}

Values are presented as mean \pm SD or number (\%).

ESRD, end stage renal disease; BMI, body mass index; SBP, systolic blood pressure; DBP, diastolic blood pressure; Cr, creatinine; eGFR, estimated glomerular filtration rate; LDL, low density lipoprotein; CRP, C-reactive protein. 